\title{
Extrinsic response enhancement at the polymorphic phase boundary in piezoelectric materials
}

\author{
Diego A. Ochoa, ${ }^{1}$ Giovanni Esteves, ${ }^{2}$ Jacob L. Jones, ${ }^{2}$ Fernando Rubio- \\ Marcos, ${ }^{3}$ José F. Fernández, ${ }^{3}$ and José E. García ${ }^{1, a)}$ \\ ${ }^{1}$ Department of Physics, Universitat Politècnica de Catalunya - BarcelonaTech, Barcelona 08034, Spain \\ ${ }^{2}$ Department of Materials Science and Engineering, North Carolina State University, Raleigh, Noth \\ Carolina 27696, USA \\ ${ }^{3}$ Department of Electroceramics, Instituto de Cerámica y Vidrio - CSIC, Madrid 28049, Spain
}

\begin{abstract}
Polymorphic phase boundaries (PPBs) in piezoelectric materials have attracted significant interest in recent years, in particular because of the unique properties that can be found in their vicinity. However, to fully harness their potential as micro-nanoscale functional entities, it is essential to achieve reliable and precise control of their piezoelectric response, which is due to two contributions known as intrinsic and extrinsic. In this work we have used a $(\mathrm{K}, \mathrm{Na}) \mathrm{NbO}_{3}$-based lead-free piezoceramic as a model system to investigate the evolution of the extrinsic contribution around a PPB. X-ray diffraction measurements are performed over a wide range of temperatures in order to determine the structures and transitions. The relevance of the extrinsic contribution at the PPB region is evaluated by means of nonlinear dielectric response measurements. Though it is widely appreciated that certain intrinsic properties of ferroelectric materials increase as PPBs are approached, our results demonstrate that the extrinsic contribution also maximizes. An enhancement of the extrinsic contribution is therefore also responsible for improving the functional properties at the PPB region. Rayleigh's law is used to quantitatively analyze the nonlinear response. As a result, an evolution of the domain wall motion dynamics through the PPB region is detected. This work demonstrates that the extrinsic contribution at a PPB may have a dynamic role in leadfree piezoelectric materials, thereby exerting a far greater influence on their functional properties than that considered to date.
\end{abstract}

a) Electronic mail: jose.eduardo.garcia@upc.edu 
The dielectric and piezoelectric responses of piezoceramics to an external stimulus, either electrical or mechanical, is due to two contributions known as intrinsic and extrinsic. The intrinsic contribution is related to the linear lattice distortion and is associated with the change in the polarization of the unit cell. This change in the polarization is related to both the polarization extension and the polarization rotation. ${ }^{1}$ The polarization rotation can be maximized in the region delimiting different polymorphic phases, such as a morphotropic phase boundary (MPB) or a polymorphic phase boundary (PPB), thereby improving macroscopic properties of materials. ${ }^{2}$ The MPB is a compositionally-driven structural change region where the coexistence of two ferroelectric phases enhances the macroscopic properties, as in the case of the $\mathrm{PbZr}_{\mathrm{x}} \mathrm{Ti}_{1-\mathrm{x}} \mathrm{O}_{3}(\mathrm{PZT})$ system. Furthermore, the PPB is a region where a temperature-driven structural change occurs, as in the case of the $\mathrm{K}_{0.5} \mathrm{Na}_{0.5} \mathrm{NbO}_{3}$ $(\mathrm{KNN})$ system. Both phase boundaries are also described as structurally bridging low symmetry regions, which have been described as monoclinic symmetry regions for both $\mathrm{PZT}^{3}$ and $\mathrm{KNN}^{4}$ systems.

The enhancement of the macroscopic properties in a PPB has recently been reported as a consequence of an electric field-induced phase transition, i.e. a polarization extension phenomenon. ${ }^{5}$ Thus, PPB is a region where material response apparently enhances due to polarization rotation and/or polarization extension. ${ }^{5,6}$ Some papers have recently emerged in which it is shown that the extrinsic effect maximizes in a MPB. ${ }^{7-9}$ In fact, Iamsasri et al. ${ }^{9}$ go further and propose that the extrinsic response enhancement may be a universal behavior in ferroelectric compositions-containing phase boundaries. Nevertheless, little evidence exists on how extrinsic effects may impact the materials properties in PPB regions.

The extrinsic contribution is easily defined as all responses different from the intrinsic one, being mainly due to domain wall motion in perovskite polycrystals. ${ }^{10}$ This contribution is considered responsible for nonlinearity, i.e. properties dependent on the applied electric 
field and/or mechanical stress, of piezoceramics. ${ }^{11}$ Thus, analysis of the nonlinear response as a function of a given parameter, e.g. temperature, composition, etc., enables the relevance of the extrinsic contribution to material response to be evaluated. The dependence of extrinsic contribution on the material properties has been well studied by using the Rayleigh model. ${ }^{12}$ This model can provide valuable information about the dynamics of domain wall motion. One hypothesis sustaining this model is that the nonlinear behavior is exclusively due to the irreversible motion of domain walls. ${ }^{13}$ Analysis of the dielectric response in terms of the Rayleigh law enables the contribution of the irreversible movement of domain walls to be studied. The reversible domain-wall motion contribution leads to deviations from the Rayleigh law predictions. ${ }^{14}$

The nonlinear contribution can be evaluated from the increments of the real $\left(\Delta \varepsilon^{\prime}\right)$ and imaginary $\left(\Delta \varepsilon^{\prime \prime}\right)$ parts of the permittivity as a function of the amplitude of the applied electric field $\left(E_{0}\right)$. The Rayleigh model assumes that the increment in the real as well as the imaginary dielectric permittivity linearly depends on the amplitude of the applied electric field $E_{0}$, as follows: ${ }^{14}$

$$
\begin{gathered}
\Delta \varepsilon^{\prime}=\alpha E_{0}, \\
\Delta \varepsilon^{\prime \prime}=\frac{4}{3 \pi} \alpha E_{0},
\end{gathered}
$$

where $\alpha$ is the Rayleigh coefficient and is directly related to the magnitude of the nonlinear response. Moreover, the ratio between the value of imaginary and real increments of dielectric permittivity is a constant that does not depend on the material:

$$
m_{\varepsilon}=\frac{\Delta \varepsilon^{\prime \prime}}{\Delta \varepsilon^{\prime}}=\frac{4}{3 \pi}=0.42
$$

Fulfillment of the relations given by Eqs. (1), (2), and (3) implies a Rayleigh behavior, associated to a preponderant irreversible motion of domain wall. In some cases, even when $\Delta \varepsilon^{\prime}$ and $\Delta \varepsilon^{\prime \prime}$ are linearly dependent on $E_{0}$, a non-Rayleigh behavior associated with a shift 
away of $m_{\varepsilon}$ from its theoretical value may take place. Nevertheless, this $m_{\varepsilon}$ shift gives us information about the preponderant mechanism in the domain wall dynamic because $m_{\varepsilon}$ relates the ratio between reversible and irreversible domain wall motion processes.

The $\left(\mathrm{K}_{0.44 \mathrm{Na}}{ }_{0.52} \mathrm{Li}{ }_{0.04}\right)\left(\mathrm{Nb}_{0.86} \mathrm{Ta}_{0.10} \mathrm{Sb}_{0.06}\right)_{3}$ (KNL-NTS) compound is used in this work as a model system to investigate the evolution of the extrinsic contribution around a PPB. KNL-NTS has been extensively studied because it is one of the most workable lead-free compositions known to date. ${ }^{15}$ The crystallographic structure of the KNL-NTS evolves from a paraelectric cubic phase $\left(P_{\mathrm{C}}\right)$ at high temperature to a ferroelectric orthorhombic phase $\left(F_{\mathrm{O}}\right)$ at low temperatures, passing through a ferroelectric tetragonal phase $\left(F_{\mathrm{T}}\right)$. The $F_{\mathrm{T}}$ to $F_{\mathrm{O}}$ phase transition gives rise to the PPB that is located close to room temperature for this compound. As a result of the existence of PPB, excellent functional properties are achieved in KNL-NTS at room temperature. ${ }^{16}$ In addition, this material shows a high nonlinear dielectric behavior at room temperature. ${ }^{17}$ Thus, studying the nonlinear behavior as a function of temperature allows the extrinsic contribution to be quantified and provides an insight into the dynamics of the domain wall movement over a wide temperature range.

The processing of the KNL-NTS is carefully described elsewhere ${ }^{18}$ and details about the microstructure are provided in the supplementary material. ${ }^{19}$ The basic properties of this compound are reported in previous works. ${ }^{17,18,20}$ X-ray diffraction (XRD) measurements were performed in a wide range of temperatures in order to effectively determine the structures and transitions. XRD studies were conducted on a PANalytical Empyrean diffractometer with a PIXcel-1D detector, which utilizes a $\mathrm{Cu} K_{\alpha}$ radiation. Measurements were completed by using two separate temperature stages: Anton Paar TTK 450 LowTemperature Chamber and Anton Paar HTK 1200. These stages enable XRD measurements from $70 \mathrm{~K}$ to $1450 \mathrm{~K}$. XRD patterns were measured in Bragg Brentano geometry from $36.5^{\circ}$ to $68.5^{\circ}$ in $2 \theta$ for low temperature measurements and from $43.5^{\circ}$ to $68.5^{\circ}$ in $2 \theta$ for high 
temperature measurements using a step size of $0.0131^{\circ}$. Low temperature measurements were made from $80 \mathrm{~K}$ to $300 \mathrm{~K}$, whereas high temperature measurements were made from $300 \mathrm{~K}$ to $1450 \mathrm{~K}$, using in both cases a ramp rate of $1 \mathrm{~K} / \mathrm{min}$. A contour plot was generated from the measured diffraction patterns by plotting all of the $1 \mathrm{D}$ intensity versus $2 \theta$ patterns sequentially. A $P_{C}$ phase and a $F_{\mathrm{O}}$ phase are detected at high and low temperatures, respectively. A $F_{\mathrm{T}}$ phase is detected at room temperature, as is expected for this material, ${ }^{18}$ indicating that the PPB is below room temperature. More details about the structure evolution of KNL-NTS are provided in the supplementary material. ${ }^{19}$

The permittivity dependence with a sub-switching ac electric field was measured at $1 \mathrm{kHz}$ by means of a capacitance comparator bridge especially designed for this type of measurement. ${ }^{21}$ In order to study the dielectric nonlinear response in temperature, the sample was placed inside a closed loop cryogenic system consisting of a helium compressor (Cryogenics 8200), a cold finger (Cryogenic model 22) and a vacuum pump (Alcatel Drytel Micro CFV100D). The system allows a sweep in a temperature range from $30 \mathrm{~K}$ to $390 \mathrm{~K}$ by using a temperature controller (LakeShore model 331). Electric field-induced polarization hysteresis loops were measured in a typical Sawyer-Tower configuration by applying a sinusoidal electric field of amplitude of $4.0 \mathrm{kV} / \mathrm{mm}$ and frequency of $1 \mathrm{~Hz}$. In this case, the sample was placed in a nitrogen bath cryostat for measurement from $200 \mathrm{~K}$ to $385 \mathrm{~K}$.

The bottom of Fig. 1 shows the real and imaginary permittivity of KNL-NTS in the temperature range from $100 \mathrm{~K}$ to $350 \mathrm{~K}$. There are no clear peaks in the permittivity, contrary to what is observed in the $P_{C}$ to $F_{\mathrm{T}}$ transition (see supplementary data ${ }^{19}$ ). Despite the lack of a sharp peak in the permittivity, some changes could be observed in both the real and the imaginary parts of the permittivity. An increase in the slope is recognized for the real part and a fairly diffuse peak appears for the imaginary part, at temperatures above $250 \mathrm{~K}$. The contour plot in the middle of Fig. 1 clearly shows the existence of the PPB region between 
$250 \mathrm{~K}$ and $300 \mathrm{~K}$, where the width and intensity of the reflection lines change when the sample goes from $F_{\mathrm{T}}$ to $F_{\mathrm{O}}$. On the other hand, the width and intensity of the reflection lines remain almost unchanged outside the PPB region (below $250 \mathrm{~K}$ and above $300 \mathrm{~K}$ ). From calculation of the intensity of the peaks from the XRD 1D-patterns for 200 reflection (top of Fig. 1), it is possible to observe that the intensity of the 002 peak remains higher than the intensity of the 200 peak at low temperatures (below $250 \mathrm{~K}$ ), as expected in the $F_{\mathrm{O}}$ phase. Otherwise, the intensity of the 200 peak is higher than the intensity of the 002 peak at high temperatures (above $300 \mathrm{~K}$ ), as expected in the $F_{\mathrm{T}}$ phase. The relative intensity switch takes place at temperatures near the PPB region, where the peak intensities reach the same value.

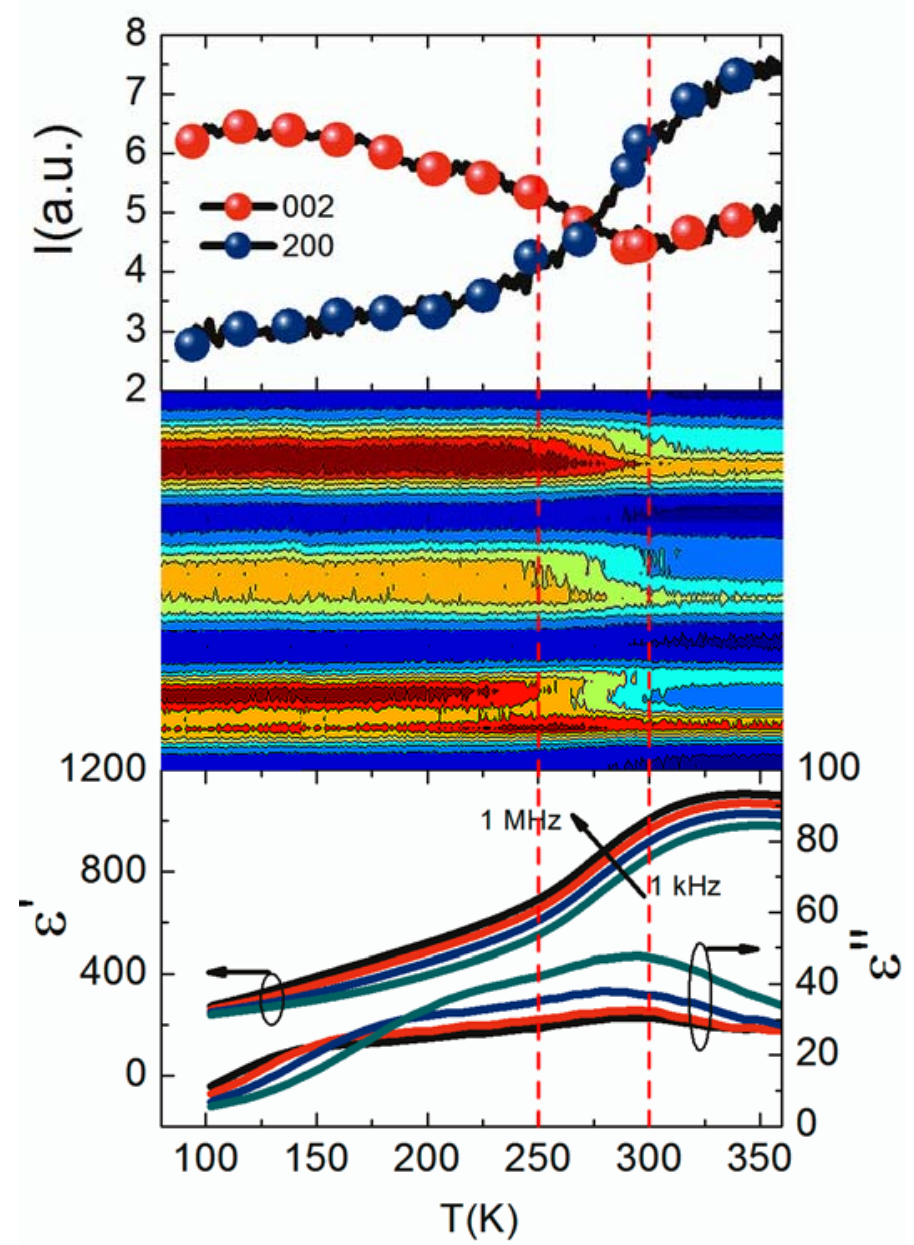

FIG. 1. Peak intensity of the 200 and 002 reflections (top), and real and imaginary parts of the permittivity at several frequencies (bottom), from low temperature $(100 \mathrm{~K})$ to above room temperature $(350 \mathrm{~K})$. A contour plot obtained from the 200, 210 and 211 reflections of the XRD patterns is shown (middle) for the same temperature range in order to reveal the PPB region. 


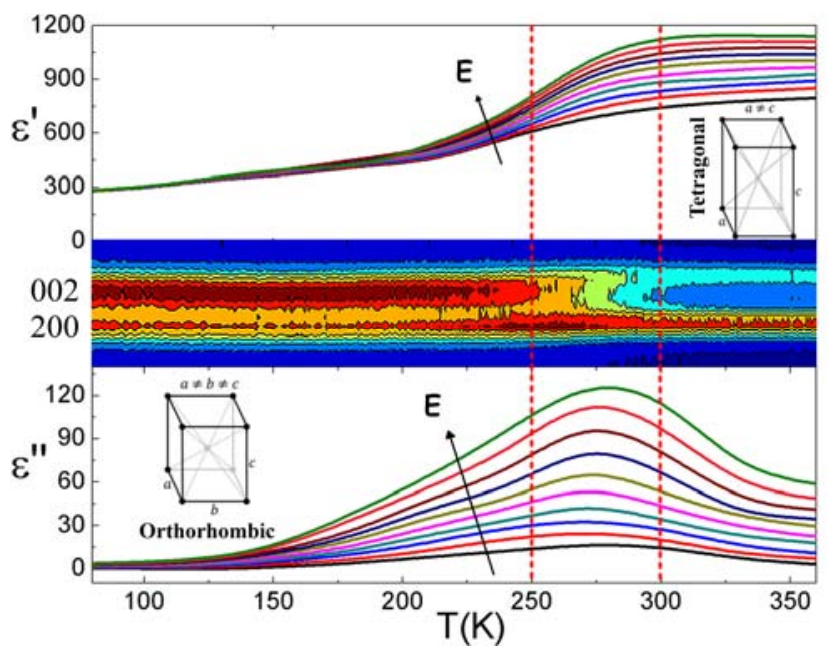

FIG. 2. Real (top) and imaginary (bottom) parts of the permittivity at different amplitudes of the applied electric field from low temperatures $(\sim 50 \mathrm{~K})$ to above $350 \mathrm{~K}$. A contour plot obtained from the 200 reflection of the XRD patterns is shown (middle) for the same temperature range. The amplitude of the electric field ranges from 0.026 to $0.26 \mathrm{MV} / \mathrm{m}$ (subswitching regime).

The dielectric response increase at the PPB region may be related to the intrinsic contribution enhancement from polarization rotation, ${ }^{1}$ but it could also be related to an increase in the extrinsic contribution. In order to analyze the role of the extrinsic contribution on properties near the PPB region, the nonlinear dielectric response, which is exclusively due to extrinsic contribution, is studied. Fig. 2 shows the real (top) and imaginary (bottom) parts of dielectric permittivity for different applied electric field amplitudes $E_{0}$, from $50 \mathrm{~K}$ to $360 \mathrm{~K}$. At very low temperatures (below $150 \mathrm{~K}$ ), the nonlinear dielectric behavior (i.e. the variation of the permittivity as a function of the applied electric field) almost disappears, as is expected from the domain wall freezing phenomenon. ${ }^{22}$ This observation proves that the nonlinear behavior is directly related to the extrinsic effect. ${ }^{11}$ The nonlinear dielectric response is significant at temperatures at which the domain wall motion contribution becomes important, usually above $150 \mathrm{~K} .{ }^{23}$ Nonlinear dielectric response increases in the $F_{\mathrm{O}}$ region as the temperature rises up to the PPB region, where the nonlinear response maximizes (this is particularly noticeable in $\varepsilon$ '), and then decreases again in the $F_{\mathrm{T}}$ region. 
It is important to point out that a nonlinear response maximum implies an extrinsic contribution maximum. The contour plot shown at the center of Fig. 2 highlights the correlation between PPB and an extrinsic response enhancement.

Fig. 3 shows the linear dependence between the increments of the imaginary $\Delta \varepsilon$ "' and the real $\Delta \varepsilon^{\prime}$ parts of the permittivity at different representative temperatures. This plot is an equivalent representation of Fig. 2, but in this case with the temperature as a parameter. Two features should be taken into account from this plot for each temperature: firstly, the distance between two adjacent points (or the length of the line connecting points) that is related to the Rayleigh coefficient $\alpha$ (Eqs. (1) and (2)), and secondly, the slope of the line connecting points that is the value of $m_{\varepsilon}$ (Eq. (3)). The length and the slope of the line connecting points evolve with the temperature. The length has a maximum at PPB region, while the slope appears to decrease as the temperature rises.

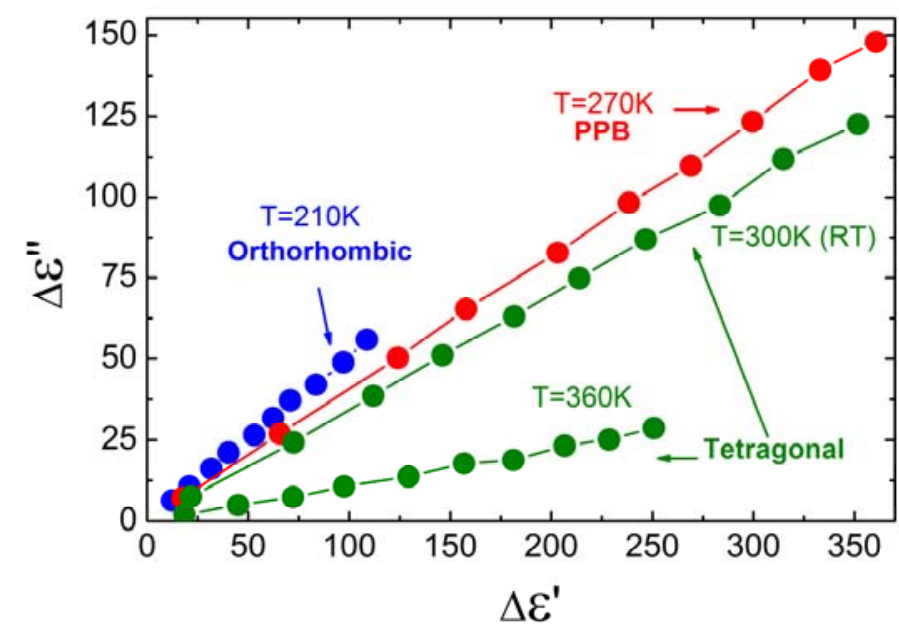

FIG. 3. Relation between the increments of the imaginary ( $\left.\Delta \varepsilon^{\prime \prime}\right)$ and real $\left(\Delta \varepsilon^{\prime}\right)$ permittivity at different representative temperatures.

A quantitative analysis of the nonlinear response can be carried out using the Rayleigh model. ${ }^{24}$ Fig. 4(a) (green points, right axis) shows the variation of the Rayleigh coefficient $\alpha$ with temperature. The value of the $\alpha$ coefficient quantifies the significance of the nonlinear effect, and then the relevance of the extrinsic contribution. The $\alpha$ value enhancement at the 
PPB region indicates an increase in the extrinsic effect as a result of the higher domain wall motions. ${ }^{25}$ Otherwise, $\alpha$ drops in both the $F_{\mathrm{T}}$ and the $F_{\mathrm{O}}$ regions, revealing that extrinsic response decreased away from the PPB. This fact may be associated to a clamp of the domain walls, as is reported in other perovskite systems for $\mathrm{MPB}^{12,13}$ and $\mathrm{PPB}^{26}$ regions. Moreover, the decrease in $\alpha$ for $F_{\mathrm{T}}$ and $F_{\mathrm{O}}$ regions is quite different according to the domain configuration of each phase. Two main domain walls appear in the tetragonal region as $90^{\circ}$ and $180^{\circ}$ domain walls. In the orthorhombic phase, in addition to the $90^{\circ}$ and $180^{\circ}$ domain walls, $60^{\circ}$ and $120^{\circ}$ domain walls are also allowable. Thus, a higher domain wall contribution is expected in the $F_{\mathrm{O}}$ region, but $\alpha$ achieves lowest values in KNL-NTS. The abrupt decrease in the $\alpha$ value in the $F_{\mathrm{O}}$ region may be understood here as a consequence of the domain-wall freezing effect. ${ }^{22}$

It is clear from the above analysis that close to the PPB region a transformation on the extrinsic contribution of the system is induced. This type of phase transition may be accompanied by the corresponding change in domain switching behavior. Electric fieldinduced polarization hysteresis loops were measured at the main $F_{\mathrm{O}}, \mathrm{PPB}$ and $F_{\mathrm{T}}$ regions, as shown in Fig. 4(b). The coexistence of orthorhombic and tetragonal domains at the PPB region promotes the polarization reorientation and, as a result, higher saturation and remanent polarizations are displayed (Fig. 4(c)). The nucleation of the orthorhombic structure is restricted by the existing tetragonal domain configuration, and the domain configuration is resolved by alternating orthorhombic and tetragonal adjacent domains. ${ }^{27,28}$ The change in the polarization direction of the orthorhombic structure converts $F_{\mathrm{T}} 180^{\circ}$ domains into non- $180^{\circ}$ domains at PPB. The domain configuration at PPB therefore allows a higher field-induced polarization because more directions are available to be oriented, and thus the material exhibits a higher irreversible extrinsic contribution. However, the internal stress due to the domain coexistence gives rise to the appearance of an unusual polarization 
relaxation at the $90^{\circ}$ domain wall, as was demonstrated in $(\mathrm{K}, \mathrm{Na}) \mathrm{NbO}_{3}$ based ceramics. ${ }^{27,28}$ At low temperatures, only the orthorhombic structure dominates and the internal stress generated diminished in accordance with the observed extrinsic response behavior.

(a)

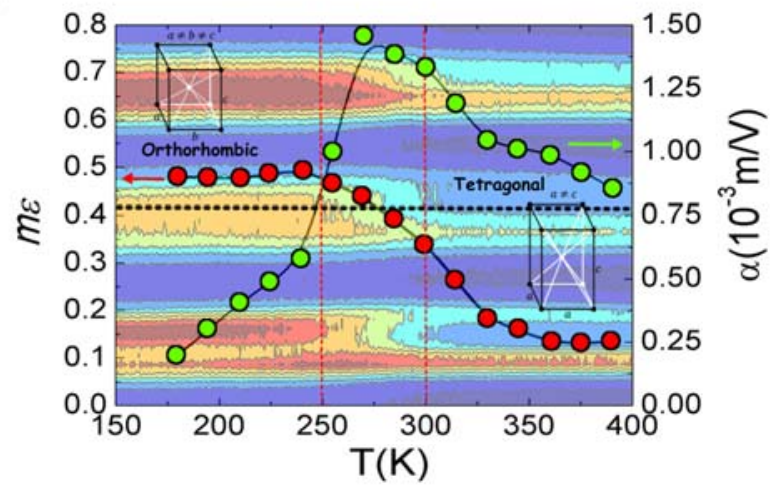

(b)

(c)

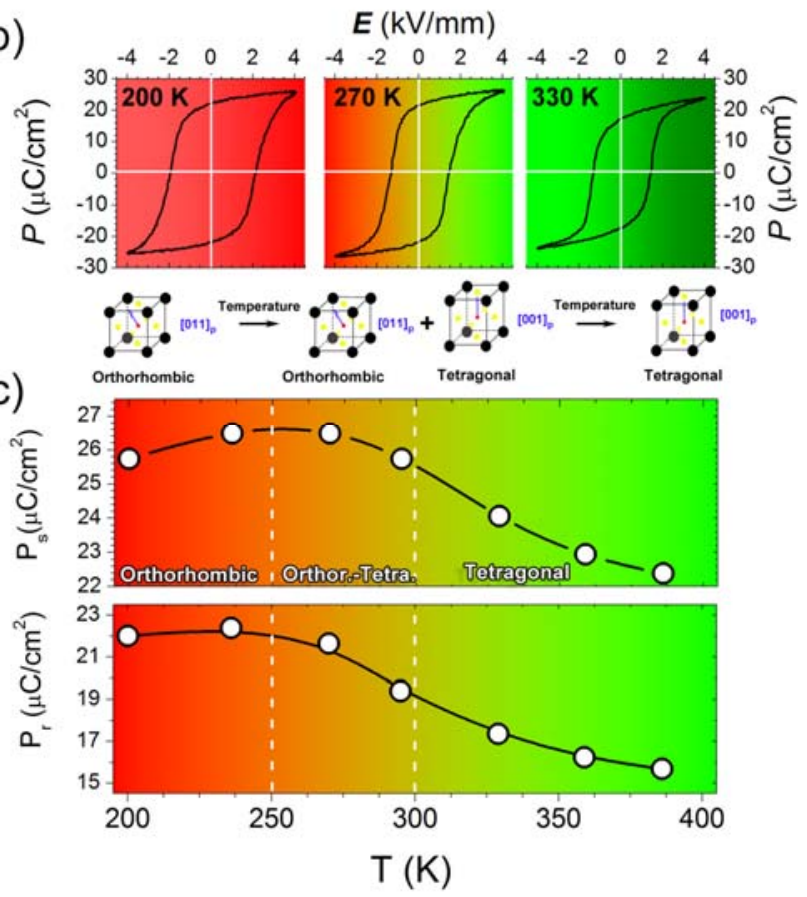

FIG. 4. (a) Rayleigh slope $m_{\varepsilon}$ (red points) and Rayleigh coefficient $\alpha$ (green points) as a function of the temperature. A contour plot obtained from the 200, 210 and 211 reflections of the XRD patterns is shown in the background of the figure in order to reveal the PPB region. The horizontal dashed line represents the $m_{\varepsilon}$ value expected from the Rayleigh model. (b) Sequence of $P-E$ loops showing the evolution of the phase transition for different temperatures localized on the main $F_{\mathrm{O}}$, PPB and $F_{\mathrm{T}}$ regions. The scheme localized at the bottom of Fig. (b) represents the schematic polarization rotation process in a perovskite unit cell $\mathrm{ABO}_{3}$, from orthorhombic phase $\left(F_{\mathrm{O}}\right)$ to tetragonal phase $\left(F_{\mathrm{T}}\right)$, where the polarizations are represented by blue arrows. (c) The saturation polarization and the remanent polarization, $P_{\mathrm{s}}$ and $P_{\mathrm{r}}$, evolution as a function of the temperature. 
Recovering Fig. 4(a), red points (left axis) show the temperature dependence of the calculated Rayleigh slope $m_{\varepsilon}$. A transition from an almost Rayleigh behavior $\left(m_{\varepsilon}\right.$ slightly above 0.42$)$ in the $F_{\mathrm{O}}$ region to a non-Rayleigh behavior $\left(m_{\varepsilon}<<0.42\right)$ in the $F_{\mathrm{T}}$ region can be observed. The $m_{\varepsilon}$ shift to lower values suggests an evolution of the dynamics of the domain walls to reversible motion. While in the $F_{0}$ region the Rayleigh model is fulfilled and the preponderant domain wall motion is irreversible, in the $F_{\mathrm{T}}$ region the $m_{\varepsilon}$ values imply an increase of the reversible domain wall motion contribution to material response. When the irreversible domain wall motion dominates the material response, a subswitching softtype behavior is displayed ${ }^{13}$, whereas when the reversible domain wall motion becomes an important contribution to the material response, a subswitching hard-type behavior emerges. ${ }^{14}$ Thus, a soft to hard behavior transition through the PPB is verified in the KNN system at the subswitching regime.

$\mathrm{A}(\mathrm{K}, \mathrm{Na}) \mathrm{NbO}_{3}$ system-based material (KNL-NTS) is used as a model for evaluating the extrinsic contribution relevance to the material properties in a polymorphic phase boundary (PPB). The PPB region is located by using X-ray diffraction in temperature. Nonlinear dielectric response measurements are carried out in order to quantify the extrinsic contribution. Results clearly show that extrinsic contribution maximizes at the PPB region. Since both intrinsic and extrinsic contributions maximize close to room temperature, excellent electromechanical properties are achieved in the KNL-NTS compound. It is for this reason that new ferroelectric systems with PPB are promising materials for specific applications in which temperature is a controlled parameter. A quantitative analysis of the nonlinear response in terms of the Rayleigh model reveals that an evolution of the domain wall motion dynamics occurs through a PPB when the temperature rises. The results thus demonstrate that the domain wall contribution must be considered when interpreting and controlling macroscopic electromechanical properties at a PPB. 
This work was supported by the MINECO (Spanish Government) project MAT201348009-C4-P. J.L.J. and G.E. acknowledge support from the National Science Foundation under award number DMR-1409399. The authors acknowledge the use of the Analytical Instrumentation Facility (AIF) at North Carolina State University, which is supported by the State of North Carolina and the National Science Foundation. F.R-M. is also indebted to MINECO for a "Juan de la Cierva'" contract (ref: JCI-2012-14521), which is co-financed with FEDER funds.

1 D. Damjanovic, Appl. Phys. Lett. 97, 062906 (2010).

2 L. Bellaiche, A. Garcia, and D. Vanderbilt, Phys. Rev. Lett. 84, 5427 (2000).

3 B. Noheda, D. E. Cox, G. Shirane, R. Guo, B. Jones, and L. E. Cross, Phys. Rev. B 63, 014103 (2000).

4 W. Ge, Y. Ren, J. Zhang, C. P. Devreugd, J. Li, and D. Viehland, J. Appl. Phys. 111, 103503 (2012).

5 T. Iamsasri, G. Tutuncu, C. Uthaisar, S. Wongsaenmai, S. Pojprapai, and J. L. Jones, J. Appl. Phys. 117, 024101 (2015).

6 J. Gao, S. Ren, L. Zhang, Y. Hao, M. Fang, M. Zhang, Y. Dai, X. Hu, D. Wang, L. Zhong, S. Li, and X. Ren, Appl. Phys. Lett. 107, 032902 (2015).

7 G. Tutuncu, B. Li, K. Bowman, and J. L. Jones, J. Appl. Phys. 115, 144104 (2014).

8 J. Gao, X. Hu, L. Zhang, F. Li, L. Zhang, Y. Wang, Y. Hao, L. Zhong, and X. Ren, Appl. Phys. Lett. 104, 252909 (2014).

9 T. Iamsasri, G. Tutuncu, C. Uthaisar, S. Pojprapai, and J. L. Jones, J. Mater. Sci. 48, 6905 (2013).

10 Q. M. Zhang, H. Wang, N. Kim, and L. E. Cross, J. Appl. Phys. 75, 454 (1994).

11 S. Li, W. Cao, and L. E. Cross, J. Appl. Phys. 69, 7219 (1991).

12 J. E. García, R. Pérez, D. A. Ochoa, A. Albareda, M. H. Lente, and J. A. Eiras, J. Appl. Phys. 103, 054108 (2008).

13 D. Damjanovic and M. Demartin, J. Phys.: Condens. Matter 9, 4943 (1997).

14 J E. Garcia, R. Perez, and A Albareda, J. Phys.: Condens. Matter 17, 7143 (2005). 
15 A. Safari, M. Abazari, K. Kerman, N. Marandian-Hagh, and E. K. Akdogan, IEEE Trans. Ultrason. Ferroelectr. Freq. Control 56, 1586 (2009).

${ }^{16}$ E. K. Akdogan, K. Kerman, M. Abazari, and A. Safari, Appl. Phys. Lett. 92, 112908 (2008).

17 D. A. Ochoa, J. A. García, R. Pérez, V. Gomis, A. Albareda, F. Rubio-Marcos, and J. F. Fernandez, J. Phys. D: Appl. Phys. 42, 025402 (2009).

18 F. Rubio-Marcos, P. Ochoa, and J. F. Fernandez, J. Eur. Ceram. Soc. 27, 4125 (2007).

19 See supplemental material for details about the structure, microstructure and high temperature dielectric properties.

${ }^{20}$ F. Rubio-Marcos, J. J. Romero, D. A. Ochoa, J. E. Garcia, R. Perez, and J. F. Fernandez, J. Am. Ceram. Soc. 93, 318 (2010).

21 J. E. García, R. Pérez, and A. Albareda, J. Phys. D. Appl. Phys. 34, 3279 (2001).

${ }^{22}$ X. L. Zhang, Z. X. Chen, L. E. Cross, and W. A. Schulze, J. Mater. Sci. 18, 968 (1983).

23 J. E. Garcia, D. A. Ochoa, V. Gomis, J. A. Eiras, and R. Pérez, J. Appl. Phys. 112, 014113 (2012).

24 D. Damjanovic and M. Demartin, J. Phys. D: Appl. Phys. 29, 2057 (1996).

25 R. E. Eitel, T. R. Shrout, and C. A. Randall, J. Appl. Phys. 99, 124110 (2006).

26 B. Peng, Z. Yue, and L. Li, J. Appl. Phys. 109, 054107 (2011).

27 F. Rubio-Marcos, A. Del Campo, R. López-Juárez, J. J. Romero, and J. F. Fernández, J. Mater. Chem. 22, 9714 (2012).

${ }^{28}$ F. Rubio-Marcos, A. Del Campo, and J. F. Fernández, J. Appl. Phys. 113, 187215 (2013). 


\title{
Extrinsic response enhancement at the polymorphic phase boundary in piezoelectric materials
}

\author{
D. A. Ochoa, G. Esteves, J. L. Jones, F. Rubio-Marcos, J. F. Fernández, and J. E. García
}

\section{Supplementary material}

The FE-SEM image shown in Figure S1a illustrates the microstructure of the KNLNTS ceramic, which was sintered at $1125{ }^{\circ} \mathrm{C}$ for $16 \mathrm{~h}$. The microstructure was evaluated using primary electrons images of field emission scanning electron microscopy (FE-SEM, Hitachi S-4700). From Figure S1a, it can be observed that the ceramic has a dense microstructure and a typical feature of quadrate shaped grains, which are common in KNN-based ceramics. A grain size distribution, GSD, of the ceramic is shown in Figure S1b. The GSD was determined from FE-SEM micrographs by an image processing and analysis program (Leica Qwin, Leica Microsystems Ltd, Cambridge, England) considering more than 200 grains in each measurement. The ceramic shows a bimodal GSD, which indicates the coexistence of two kinds of grain population. The population associated to small grain is centred at $1.4 \mu \mathrm{m}$, while the population corresponding to the larger grain is localized to 3 $\mu \mathrm{m}$. EDS analysis was carried out to verify the composition of the KNL-NTS ceramic (Figure S1c). The EDS analysis indicated that the KNL-NTS ceramic (area marked as 1 in Fig. S1a) has the typical nominal composition with a $\mathrm{Na} / \mathrm{K}$ concentration ratio of around 1.16, slightly lower than the nominal ratio of 1.18 (see Table 1 in Fig. S1). 
a

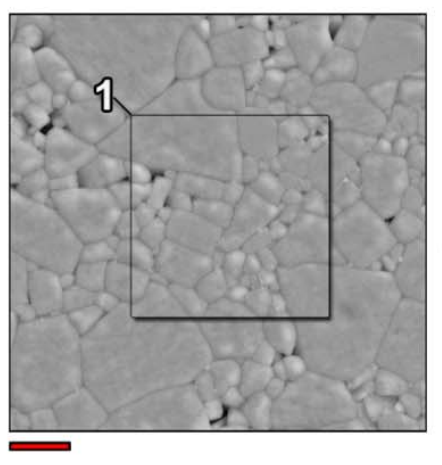

b

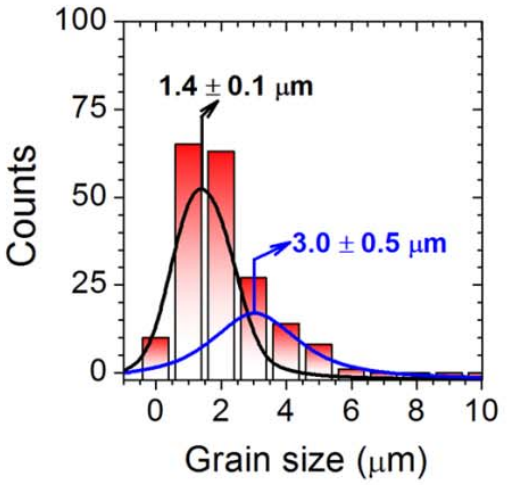

C

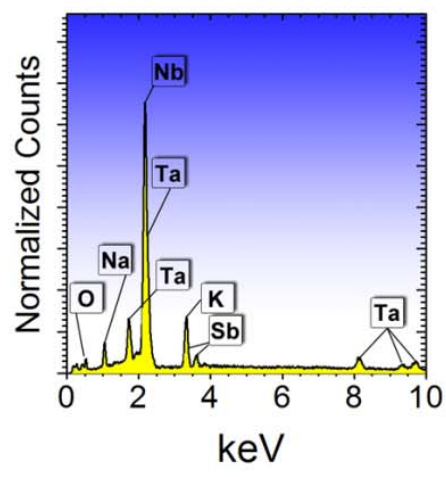

Table 1. The table presents the composition on the area shown on micrograph of the panel a derived from EDS spectra. The table represents the atomic percentages of elements.

\begin{tabular}{lcccccccc}
\hline & $\mathrm{O}$ & $\mathrm{Na}$ & $\mathrm{K}$ & $\mathrm{Li}$ & $\mathrm{Nb}$ & $\mathrm{Ta}$ & $\mathrm{Sb}$ & $\mathrm{Na} / \mathrm{K}$ \\
\hline Area 1 & 60.44 & 9.91 & 8.50 & -- & 18.20 & 2.19 & 0.76 & $\mathbf{1 . 1 6}$ \\
KNL-NTS $_{\text {nominally composition }}$ & 60.00 & 10.40 & 8.80 & 0.80 & 17.20 & 2.00 & 0.80 & $\mathbf{1 . 1 8}$ \\
\hline
\end{tabular}

Supplementary Figure S1 | Microstructural characterization of the KNL-NTS ceramic: Panel a shows the microstructure of polished and thermally etched surface of KNL-NTS sintered ceramic at $1125^{\circ} \mathrm{C}$ during 16 hours. The sample has a dense microstructure with cuboidal shaped grains, which is a common feature in KNL-NTS based ceramics. Scale bar, $5 \mu \mathrm{m}$. Panel $\mathbf{b}$ shows the grain size distribution (GSD) of KNL-NTS ceramic. The GSD is fitted to the sum of two Gaussian peaks because of the system presents a bimodal distribution. Panel (c) shows the EDS spectrum corresponding to the area marked as 1 in panel a. Table 1 shows the composition derived from EDS spectra, which represents the atomic percentages of elements. 
Figure S2 shows XRD 1D-patterns at three representative temperatures. As the structure goes from a higher symmetry phase to a lower symmetry phase, paraelectric cubic $\left(P_{\mathrm{C}}\right)$ to ferroelectric tetragonal $\left(F_{\mathrm{T}}\right)$, the amount of degenerate reflections increases. It is possible to observe the change in the relative intensity of the peaks in the 200 reflection between ferroelectric orthorhombic $\left(F_{\mathrm{O}}\right)$ and $F_{\mathrm{T}}$. At low temperature, the 002 peak intensity is higher than the 200 peak, while the most intense is the 200 peak at room temperature, as is expected for $F_{\mathrm{O}}$ and $F_{\mathrm{T}}$ phases, respectively. The discrepancy between the intensity of the 200 reflections between phases is due to the differences in structure factor, as different phases have different atomic positions.

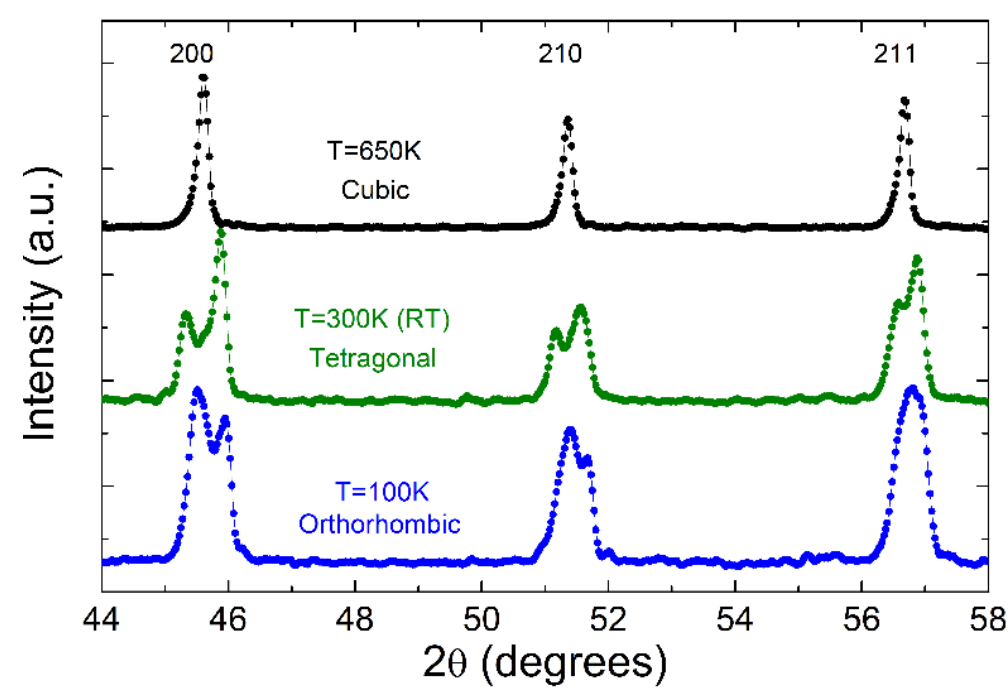

Supplementary Figure S2 | X-ray diffraction 1D-patterns at different representative temperatures: The different crystallographic phases of the KNL-NTS ceramic are displayed. A cubic phase and an orthorhombic phase are detected at high and low temperatures, respectively, while a tetragonal phase is detected at room temperature. 
The bottom of Figure S3 shows the temperature dependence of the real and imaginary parts of the permittivity, at several frequencies, in the region from room temperature to high temperature. A precision LCR meter (Agilent E4980A) is used to obtain the permittivity while the sample is appropriately placed in a tubular oven. Both real and imaginary parts of the permittivity display a maximum at 570 $\mathrm{K}$ that corresponds to the $P_{\mathrm{C}}$ to $F_{\mathrm{T}}$ second order phase transition. The phase transition can be verified in the contour plot from the XRD pattern versus temperature (top of Figure S3) and shows the split in the different reflections when the temperature decreases, revealing the presence of a lower symmetry phase. The changes in the diffraction intensities match well with changes in the dielectric properties. Below that transition, from $500 \mathrm{~K}$ to room temperature, the measured permittivity values flatten out exhibiting stable dielectric properties.

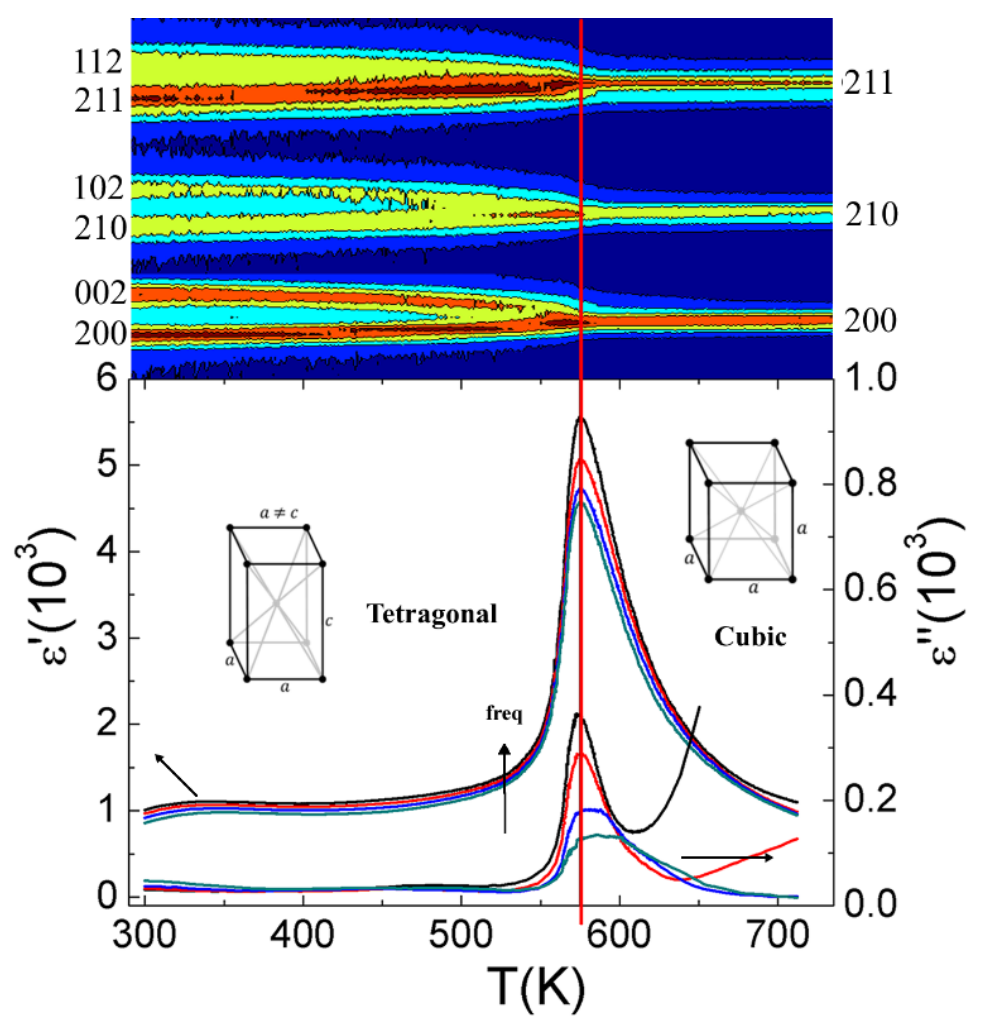

Supplementary Figure S3 | Contour plot obtained from the XRD patterns (top) and real and imaginary parts of the permittivity at several frequencies (bottom): The changes in the diffraction intensities as well as the peak in the dielectric properties correspond to the paraelectric to ferroelectric second order phase transition. Measurements were performed from room temperature to above $700 \mathrm{~K}$. 
This is the post-print (i.e. final draft post-refereeing) of the publication.

The final publication is available at Elsevier via http://dx.doi.org/10.1063/1.4945593 